\title{
THE IMPORTANCE OF HELIUM AND METALS DIFFUSION IN STARS
}

\author{
CHARLES R. PROFFITT \\ Computer Sciences Corporation and Catholic University of \\ America, Code 680, Goddard Space Flight Center, Greenbelt \\ MD 20771, USA
}

\begin{abstract}
Comparisons between models of the solar interior and sound speed profiles derived from inversions of helioseismic data have demonstrated that it is essential to include the effects of gravitational settling when calculating the structure and evolution of the Sun. Including settling should also be necessary for models of metal poor main-sequence stars and results in a substantial reduction in the ages derived for globular clusters.

In many cases it is clear that competing hydrodynamic processes, such as mass loss or rotationally driven mixing, will limit the effectiveness of gravitational separation of chemical elements. However, the quantitative details and even the relative importance of the different processes in various types of stars remains poorly understood.
\end{abstract}

\section{Introduction}

The chemical separation of elements in stellar atmospheres and interiors is driven by gravitational and radiative forces and is a basic physical process which should not be ignored in computations of stellar evolution. Preventing chemical separation requires the existence of competing hydrodynamic mechanisms, such as mixing or mass loss, which may have substantial consequences of their own for a star's structure and evolution.

The effects of diffusion are most obvious in white dwarfs and the chemically peculiar A and B stars, where extraordinarily large abundance anomalies occur. Detailed calculations are difficult for these stars because of the short timescales involved and the large radiative acceleration expected for the individual elements. In this review we will concentrate on the more

T.R. Bedding et al. (eds.),

Fundamental Stellar Properties: The Interaction between Observation and Theory, 355-360.

(C) 1997 IAU. Printed in the Netherlands. 
subtle effects of diffusion on the interiors of low-mass main-sequence stars, especially the Sun and main-sequence metal-poor dwarfs.

\section{Physics of Diffusion}

Most work on chemical diffusion uses either the Chapman-Enskog procedure (Chapman \& Cowling 1970) or the method of Burgers (1969) for deriving the transport properties from the Boltzmann equation. The method of Burgers is more easily generalized to multi-component plasmas, and has been adapted for the case of partially ionized plasmas by Geiss \& Burgi $(1986,1987)$. For collisions between charged particles, the collision integrals computed numerically by Paquette et al. (1986) which assume screened Coulomb potentials are currently the most reliable. Caution should be used when substituting approximate analytic expressions.

How reliable are current estimates of diffusion rates? It should be noted that many of the assumptions implicit in the diffusion calculations are only marginally satisfied in the interiors of main-sequence stars (see the discussion in Michaud \& Proffitt 1993). The Coulomb interaction energies of colliding particles are only a few times smaller than their kinetic energies, and there are typically only a few screening particles per Debye sphere. The effects of inelastic collisions on diffusion coefficients in ionization zones have also not been considered. While there is no reason to believe that any of these effects cause serious errors in calculations of the transport properties, a derivation done in the context of an equation of state which rigorously treats multi-particle interactions, such as the OPAL EOS (Rogers 1994), would be extremely valuable. In the absence of such work, it is difficult to assign quantitative errors to current treatments. It does seem likely that the stronger Coulomb coupling and more complicated electronic structures of heavy elements would result in uncertainties in diffusion rates substantially larger than for hydrogen and helium.

\section{The Sun}

In a star like the Sun, gravitational settling of the helium and metals will be the primary effect of atomic diffusion. Noerdlinger (1977) first studied the effects of the gravitational settling of helium in solar models, and found that surface helium decreased by a few percent over the lifetime of the Sun, and that there was a slight acceleration of the core evolution caused by central settling. These results were mostly ignored for a number of years. However, more recent work done with more accurate diffusion coefficients and more carefully matched to the observed solar parameters finds similar results (Cox, Guzik, \& Kidman 1989, Proffitt \& Michaud 1991, and Bahcall \& Pinsonneault 1992 among others). These latter works find that the 
current mass fraction of helium in the solar convection zone has decreased by about $10 \%,(\Delta Y \approx-0.03)$, since the Sun formed. Models with gravitational settling also have slightly deeper convection zones and slightly more concentrated cores. There is also a steep gradient in the helium abundance just below the surface convection zone. Guzik et al. (1989), Proffitt (1994), and Bahcall \& Pinsonneault (1995) also considered the effects of heavy element settling on solar structure, and found them smaller than the effects of helium settling alone, but still significant.

As discussed elsewhere in these proceedings (see the contribution by Christensen-Dalsgaard) helioseismology can be used to test models of the structure of the solar interior. Because of uncertainties in the superficial layers of the solar convection zone, comparison between theory and observation is best done by comparing the sound speed profile of computed models with a seismic model derived from an inversion of the observed frequencies (Christensen-Dalsgaard, Gough, \& Thompson 1989). Inversion techniques were first used to test models that included helium settling by ChristensenDalsgaard, Proffitt, and Thompson (1993). Additional comparisons using improved input physics and observational data have recently been done by Basu et al. (1996), Antia (1996), and Richard et al. (1996). They all find that models with settling reproduce the interior sound speed of the Sun to within $0.5 \%$ throughout the region where the observations are reliable, and are clearly superior to models which neglect gravitational settling. There is a suggestion that the calculated helium abundance gradient just below the convection zone is steeper than in the real Sun. This suggests that some modest amount of mixing has occurred below the solar convection zone, and is consistent with the mixing required to explain the observed solar lithium depletion. There are also some hints that the Sun's central helium profile is slightly flatter than that of the models, which might be evidence for a small amount of mixing in the core. However, it is not possible to unambiguously distinguish errors in the abundance profile from errors in the opacities.

Helioseismology observations can also be used to measure the helium abundance in the surface convection zone. By measuring the sound speed as a function of depth in the second helium ionization zone, the helium mass fraction can be inferred, provided the equation of state is sufficiently well understood. This has been done recently by Dziembowski et al. (1994), Hernandez \& Christensen-Dalsgaard (1994), Basu \& Antia (1995), and Richard et al. (1996). They find $Y \approx 0.24-0.25$ in the surface convection zone, which is substantially less than the initial helium abundance, $Y \approx 0.27-$ 0.28 , needed to produce stellar interior models that match the current solar luminosity. This provides additional strong evidence that the predicted amount of helium settling has occurred. 


\section{Metal Poor Dwarfs}

The material in the interiors of the metal poor $\mathrm{G}$ and $\mathrm{K}$ main-sequence and turn-off stars of the halo and the globular clusters has densities and temperatures similar to that of the solar interior. Since we appear to understand the equation of state, nuclear reaction rates, opacities, and diffusion rates well enough to build extremely accurate solar models, it might seem unlikely that there is any new physics needed to model these stars. The most significant difference is that many of these stars have substantially thinner surface convection zones than the Sun. This is especially true for the most metal-poor turn-off stars with $T_{\text {eff }} \approx 6000$ to $6400 \mathrm{~K}$.

The effects of helium settling on the evolution of low mass globular cluster stars was first studied by Stringfellow et al. (1983), who found that settling in the center of the star accelerated the main-sequence evolution, thereby reducing the turn-off luminosity at a given age. From comparisons of calculated evolutionary tracks they suggested that this would reduce the estimated ages for globular clusters by $25 \%$. However, newer studies using improved physics and, more importantly, directly comparing isochrones rather than evolutionary tracks (e.g., Proffitt \& VandenBerg 1991), found that including helium diffusion reduces globular cluster ages as deduced from the luminosity of the main-sequence turn-off by about $10 \%$. Ages estimated from the difference in luminosity between the turn-off and zero age horizontal-branch (ZAHB) (e.g. Chaboyer, Sarajedini, \& Demarque 1992) are less affected, because helium settling in the interior results in a surface helium mass fraction after the first dredge up that is slightly lower than in models without diffusion. This leads to lower predicted ZAHB luminosities, partially offsetting the decrease in the turn-off luminosity. However, Sweigart (1997) has recently suggested that extra helium from the shell source might be mixed into the envelope during the first ascent RGB, increasing the luminosity of the ZAHB and decreasing the derived ages for globular clusters. This suggests that any theoretical calculation of the ZAHB luminosity should be avoided in measuring absolute ages for globular clusters. As noted above, calculations of main-sequence evolution can be tested using the Sun, and therefore observations of the turn-off and sub-giant luminosities combined with measures of the distance moduli not based on theoretical HB luminosities appear to be the most reliable way to measure the absolute ages of globular clusters.

It is, however, also clear that simple, uninhibited helium settling does not occur in the surface layers of metal poor dwarfs. Models with a reduced surface helium abundance have larger radii and cooler $T_{\text {eff }}$. Proffitt \& VandenBerg (1991) found that the morphology of such isochrones is inconsistent with the observed color-magnitude diagrams of metal poor globular clusters 
such as M92. Helium settling is also accompanied by a similar reduction in the lithium abundance (which sinks at almost the same rate as helium), and this would lead to noticeable lithium depletion in the warmest halo turn-off stars which have the thinnest convective envelopes. However, observations have consistently shown no trace of this "Li dip", and instead suggest a slight increase of the lithium abundance with increasing $T_{\text {eff }}$ (see Spite $e t$ al. 1996, and Spite 1997 in this volume).

The surface settling of lithium (and helium) must be moderated in such a way that a nearly uniform abundance is maintained in most mainsequence halo stars with $T_{\text {eff }}$ between 6500 and $5700 \mathrm{~K}$. Swenson (1995) and Vauclair \& Charbonnel (1995) have demonstrated that models combining gravitational settling with mass loss rates of a few $\times 10^{-13} M_{\odot} \mathrm{yr}^{-1}$ can match the observed abundance pattern, but it is unclear if such a large and uniform mass loss rate is plausible, (the solar mass loss rate is $\left.10^{-14} M_{\odot} \mathrm{yr}^{-1}\right)$. Mixing processes related to rotation have also been proposed as a way to limit settling (e.g., Vauclair 1988, Chaboyer 1994). Preventing too much settling in the warm stars implies that at least the outer 1 to $2 \%$ of the stellar envelopes are well mixed, even in stars with surface convection zones much thinner than this. However, mixing that extends much beyond the outer $2 \%$ would be inconsistent with the apparent detection of ${ }^{6} \mathrm{Li}$ in at least one near turn-off halo star (Smith, Lambert, \& Nissen 1994), as ${ }^{6} \mathrm{Li}$ is more easily destroyed by nuclear reactions than the more abundant isotope ${ }^{7} \mathrm{Li}$. Mixing beyond the outer $3 \%$ of the mass would begin to destroy significant amounts of ${ }^{7} \mathrm{Li}$.

\section{Conclusions and Recommendations}

We must reconcile the helioseismic observations of the Sun, which show that the the expected helium settling has been at most slightly moderated by mixing below the surface convection zone, with the lithium abundances observed in the halo stars, which suggest that the depth of surface mixing is independent of the depth of the surface convection zone. The observed lithium abundances strongly suggest that the mixed region in the globular cluster/halo stars is shallower than in the Sun. Since settling has had three times longer to act in these stars, it seems inevitable that a significant amount of helium settling has occurred. The surface helium mass fraction in these stars is likely to be in the range 0.1-0.2 rather than at the expected initial abundances of $0.23-0.25$. This should cause a small redward shift $(\approx 0.02$ to 0.03 in $B-V)$ in the predicted location of the $\mathrm{F}$ and early G-stars relative to the RGB, although uncertainties in convection theory and spectral synthesis models may prevent direct detection of this effect.

We strongly recommend that all future isochrone calculations for 0.6- 
$1.2 M_{\odot}$ stars include the gravitational settling of helium and heavy elements, along with a model of some other hydrodynamic process which prevents excessive settling that is inconsistent with the observed chemical abundances of lithium and other elements. The physics used in such calculations should always be checked against precise solar seismic models. It is probably premature to identify any particular mixing or mass-loss formulation as the mechanism that limits settling, and this regrettably limits the usefulness of parameter-free diffusion models. However, at least for metal poor dwarfs, available data places strong limits on amount of surface settling that has occurred, and this should enable models to be produced that are significantly superior to any that ignore gravitational settling.

\section{References}

Antia, H. M. 1996, $A \& A$, 307, 609

Bahcall, J. N. \& Pinsonneault, M. H. 1992, ApJ, 395, 119

Bahcall, J. N. \& Pinsonneault, M. H. 1995, Rev. Mod. Phys., 67, 781

Basu, S. \& Antia, H. M. 1995, MNRAS, 276, 1402

Basu, S, Christensen-Dalsgaard, J., Schou, J., Thompson, M. J., \& Tomczyk, S. 1996, ApJ, 460, 1064

Burgers, J. M. 1969, Flow Equations for Composite Gases, New York: Academic Press

Chaboyer, B. 1994, PASP, 106, 200

Chaboyer, B., Sarajedini, A., \& Demarque, P. 1992, ApJ, 394, 515

Chapman, S. \& Cowling, T. G., 1970 The Mathematical Theory of Non-Uniform Gases, Cambridge University Press, 3rd ed.

Christensen-Dalsgaard, J., Gough, D. O., \& Thompson, M. J. 1989, MNRAS, 238, 481

Christensen-Dalsgaard, J. Proffitt, C. R. \& Thompson, M. J. 1993 ApJ, 403, 75

Cox, A. N., Guzik, J. A., \& Kidman, R. B 1989, ApJ, 342, 1187

Dziembowski, W. Q., Goode, P. R., Pamyatnikh, A. A., \& Sienkiewicz, R. 1994, ApJ, 432, 417

Geiss, J. \& Bürgi, A. 1986, $A \varangle A, \mathbf{1 5 9}, 1$

Geiss, J. \& Bürgi, A. 1987, $A \mho A, 178,286$

Hernandez, F. P., \& Christensen-Dalsgaard, J. 1994, MNRAS, 269, 475

Michaud, G. \& Proffitt, C. R. 1993, in Inside the Stars, eds. W. W. Weiss, A. Baglin, IAU Coll. 137, PASP Conf. Ser. 40, 246

Noerdlinger, P. D. 1977, $A \& A, \mathbf{5 7}, 407$

Paquette, C., Pelletier, C., Fontaine, G., \& Michaud, G. 1986, ApJ, 61, 177

Proffitt, C. R. 1994, ApJ, 425, 849

Proffitt, C. R. \& Michaud, G. 1991, ApJ, 380, 238

Proffitt, C. R. \& VandenBerg, D. A. 1991, ApJS, 77, 473

Richard, O., Vauclair, S., Charbonnel, C., Dziembowski, W. A. 1996, $A \& A, \mathbf{3 1 2}, 1000$

Rogers, F. J. 1994, in IAU Coll. 147, The Equation of State in Astrophysics, ed. G. Chabrier, \& E. L. Schatzman, Cambridge, Cambridge University Press, 16

Smith, V. V., Lambert, D. L., \& Nissen, P. E. 1993, ApJ, 408, 262

Spite, M., Francois, P., Nissen, P. E., \& Spite, F. 1996, A\&A, 307, 172

Stringfellow, G. S., Bodenheimer, P., Noerdlinger, P. D., \& Arigo, R. J. 1983, ApJ, 264, 228

Sweigart, A. V. 1997, ApJL, 474, 23

Swenson, F. J. 1995, ApJL, 438, 87

Vauclair, S. 1988, ApJ, 335, 971

Vauclair, S. \& Charbonnel, C. 1995, A\&A, 295, 715

Discussion of this paper appears at the end of these Proceedings. 